Ergod. Th. \& Dynam. Sys. (1990), 10, 101-117

Printed in Great Britain

\title{
Suspensions of topological transformation groups
}

\author{
DAVID B. ELLIS \\ Beloit College Box 82, Beloit WI 53511, USA \\ (Received 30 October 1987 and revised 5 December 1988)
}

\begin{abstract}
Let $S$ be a subgroup of a topological group $T$, and suppose that $S$ acts on a space $X$. One can form a $T$-transformation group $\left(X \times_{S} T, T\right)$ called the suspension of the $S$-transformation group $(X, S)$. In this paper we study the relationship between the dynamical properties of $(X, S)$ and those of its suspension when $S$ is syndetic in $T$. The main tool used in this study is a notion of the group of a minimal flow $(X, T)$ which is sensitive to the topology on the group $T$. We are able, using this group and the enveloping semigroup to obtain results on which $T$ transformation groups can be realized as suspensions of $S$-transformation groups, and give conditions under which the suspension of an equicontinuous $S$-flow is an equicontinuous $T$-flow.
\end{abstract}

\section{Introduction}

When $S$ is a closed subgroup of a topological group $T$ and $(X, S)$ is an $S$ transformation group one can form the suspension $X \times_{S} T$ of $(X, S)$ to a $T$ transformation group. Two natural questions arise: what is the relationship between the dynamical properties of $(X, S)$ and those of $X \times_{S} T$ ? and which $T$-transformation groups are suspensions of $S$-transformation groups? In this paper we obtain some answers to these questions under the assumption that $S$ is a closed normal syndetic subgroup of $T$. For example we show that equicontinuity is not in general preserved under suspension and give conditions on $S$ and $T$ under which it is. We also obtain a complete answer to the second question.

The main tool used is the group of a minimal flow which is described in $\S 3$. Here we give a definition of the group of a minimal flow which is sensitive to the topology on the group $T$; this group is the analogue of the group defined by $\mathrm{R}$. Ellis (see for example [E1]) and the two coincide when $T$ is given the discrete topology. In particular in $\S 4$ we describe conditions in terms of this group which are necessary and sufficient for $(X, T)$ to be the suspension of an $S$-flow. When $T$ is abelian we also obtain conditions under which $(X, T)$ is totally minimal.

The paper is organized into five sections. In the first we recall some preliminaries and set our notation. The second section contains a few elementary properties of suspensions, and an example of an equicontinuous flow whose suspension is not 
equicontinuous. In $\S 3$ the group of a minimal flow is defined and some of its properties deduced. The results in $\S 3$ are used in $\S \S 4$ and 5 to study the question of realizing flows as suspensions and equicontinuity of suspensions respectively.

\section{Preliminaries}

In this section we set notation and mention some of the results we will need to use in the following sections. For further details and proofs see [E2].

We begin with some standard definitions regarding topological transformation groups.

Definition 1.1. A topological transformation group (flow) is a pair $(X, T)$ where $X$ is a compact Hausdorff space and $T$ is a locally compact topological group, together with a continuous map

$$
\begin{gathered}
X \times T \rightarrow X \\
(x, t) \rightarrow x t .
\end{gathered}
$$

We say that $(X, T)$ is pointed if $X$ is provided with a base point $x_{0}$, such that the orbit closure of $x_{0}$ is $X$, that is

$$
\overline{x_{0} T}=\overline{\left\{x_{0} t / t \in T\right\}}=X
$$

A homomorphism of pointed flows $(X, T) \rightarrow(Y, T)$ is a continuous map $\varphi: X \rightarrow Y$ such that $\varphi\left(x_{0}\right)=y_{0}$ and $\varphi(x t)=\varphi(x) t$ for all $t \in T$ and $x \in X$. Note that if a homomorphism exists it is unique. We often refer to $(Y, T)$ as a factor of $(X, T)$ or to $(X, T)$ as an extension of $(Y, T)$.

Let $(X, T)$ be a flow and $A \subset X$, we say that $A$ is a minimal set if $\overline{x T}=A$ for all $x \in A$. If $X$ itself is a minimal set we refer to $(X, T)$ as a minimal flow.

Definition 1.2. Let $(X, T)$ be a pointed transformation group, and denote by

$$
X^{X}=\{f: X \rightarrow X\}
$$

the set of all functions from $X$ to $X$. We give $X^{X}$ the topology of pointwise convergence and define the enveloping semigroup of $(X, T)$ by

$$
E(X, T)=\bar{T} \subset X^{X} \text {. }
$$

Note that $T \subset X^{X}$ since each element of $T$ acts as a homeomorphism on $X$. Moreover $X^{X}$ and hence $E(X, T)$, is a semigroup under composition of functions. In fact $E(X, T)$ is a pointed flow.

We will need some facts about $E(X, T)$.

Proposition 1.3. Let $(X, T)$ be a minimal transformation group. Then

(1) $(X, T)$ is distal if and only if $E(X, T)$ is a group

(2) $(X, T)$ is equicontinuous if and only if $E(X, T)$ is a group of homeomorphisms.

Remarks 1.4. (1) We recall that by definition $(X, T)$ is distal if for every $x \neq y \in X$,

$$
\begin{aligned}
\varnothing & =\overline{(x, y) T} \cap \Delta \\
& =\overline{\{(x t, y t) \in X \times X / t \in T\}} \cap\{(z, z) / z \in X\} .
\end{aligned}
$$


(2) By definition $(X, T)$ is equicontinuous if $T$ is an equicontinuous family of homeomorphisms of $X$.

\section{Suspensions of topological transformation groups}

In this section we will define the suspension of an $S$-transformation group to a $T$-transformation group and discuss some elementary properties of this construction.

From now on $T$ will denote a locally compact topological group and $S \subset T$ a closed syndetic subgroup given the induced topology. Recall that $S$ is a syndetic subgroup of $T$ if there exists a compact subset $K \subset T$ with $S K=T$.

Definition 2.1. Let $S \subset T$ be a closed syndetic subgroup of $T$ and $(X, S)$ an $S$ transformation group. We define an action of $S$ on $X \times T$ by

$$
s(x, t)=\left(x s^{-1}, s t\right)
$$

Then the suspension of $(X, S)$ is given by

$$
\underset{S}{X} T=X \times T / \sim,
$$

where $\left(x_{1}, t_{1}\right) \sim\left(x_{2}, t_{2}\right)$ iff $s\left(x_{1}, t_{1}\right)=\left(x_{2}, t_{2}\right)$ for some $s \in S$.

We will denote the equivalence class of $(x, t)$ by $[x, t]$. We observe that $X \times_{s} T$ is a compact Hausdorff space because $S$ is closed and syndetic. The map

$$
\begin{gathered}
X \times T \times T \rightarrow \underset{S}{X} \underset{S}{X} \times T \\
\left([x, t], t_{1}\right) \rightarrow\left[x, t t_{1}\right],
\end{gathered}
$$

defines a continuous action of $T$ on $X \times_{\mathrm{S}} T$.

We will be interested in which properties of the $S$-transformation group $(X, S)$ are preserved by suspension. The proofs of the next two lemmas are straightforward and will be left to the reader.

Lemma 2.2. Let $\varphi:(X, S) \rightarrow(Y, S)$ be a homomorphism of $S$-transformation groups. Then $\varphi$ induces a homomorphism $\hat{\varphi}: X \times_{S} T \rightarrow Y \times_{S} T$ of T-transformation groups.

LEMMA 2.3. Let $(X, S)$ be an $S$-transformation group. Then

(1) If $\overline{x_{0} S}=X$ then $\overline{\left[x_{0}, e\right] T}=X \times_{s} T$.

(2) If $(X, S)$ is minimal then $X \times_{S} T$ is minimal.

(3) If $(X, S)$ is distal then $X \times_{S} T$ is distal.

Proposition 2.4. Let $S$ be a closed syndetic subgroup of $T$ which is contained in the center of $T$. Let $(X, S)$ be an equicontinuous $S$-transformation group. Then $X \times_{S} T$ is an equicontinuous $T$-transformation group.

Proof. It suffices to show that the $S$-transformation group $\left(X \times_{S} T, S\right)$ is equicontinuous. Now since $S$ is syndetic in $T$ there exists a compact subset $K \subset T$ with $K S=T$. We form the $S$-transformation group $(X \times K, S)$ using the action

$$
(x, k) s=(x s, k) \text {. }
$$


It is clear that the assumption that $(X, S)$ is equicontinuous implies that $(X \times K, S)$ is equicontinuous. On the other hand, the map

$$
\begin{aligned}
(X \times K, S) & \rightarrow \underset{s}{(X \times T)} \\
(x, k) & \rightarrow[x, k],
\end{aligned}
$$

is an epimorphism of $S$-transformation groups and hence $\left(X \times_{S} T, S\right)$ is equicontinuous.

Remarks 2.5. (1) When $T$ is given the discrete topology the assumption that $S$ is contained in the center of $T$ can be replaced by the weaker assumption that $S$ is normal in $T$. The proof needs to be modified slightly because in this case the map $(X \times K, S) \rightarrow\left(X \times{ }_{s} T, S\right)$ given above need not be a homomorphism. Nevertheless the result still follows because the compact subset $K$ of $T$ must be finite.

(2) For a general topology on $T$ it is possible to have a closed syndetic normal subgroup $S$ of $T$ where $(X, S)$ is equicontinuous but $\left(X \times_{S} T, T\right)$ is not. The following example illustrates this fact.

Example 2.6. Let $S=\mathbf{R}^{2}$ (the Lie group under addition). Then the circle $S^{1}$ acts on $S$ by rotations and we can form the semidirect product

$$
T=S \rtimes S^{1}
$$

( $T$ is simply a subgroup of the group of rigid motions in $\mathbf{R}^{2}$.) Then $T$ is a connected locally compact solvable Lie group and $S$ is a syndetic normal subgroup of $T$.

Set $X=S^{1} \times S^{1}$, the torus. Then the standard action of $S=\mathrm{R}^{2}$ on $X$ by covering transformations factors through the action of $X$ on itself by multiplication and hence is equicontinuous.

We claim that $T$ does not act equicontinuously on $X \times_{s} T$. To see this first note that $T$ acts effectively on $X \times_{s} T$. This is because if $[x, t] t_{1}=[x, t]$ for all $t \in T$ then $t_{1} \in S$ and $t t_{1} t^{-1}$ acts trivially on $X$ for all $t \in T$. It is easy to check that this implies that $t_{1}=e$. The fact that $T$ does not act equicontinuously on $X \times_{S} T$ now follows from the fact that any connected locally compact solvable group which acts effectively and equicontinuously on a compact Hausdorff space is abelian. This fact is a corollary of the following lemma.

This example was motivated by [Mc-I-W].

Leмma 2.7. Let $T$ be a connected locally compact solvable topological group. Let $(X, T)$ be an equicontinuous $T$-transformation group. Then the commutator subgroup of $T$ acts trivially on $X$.

Proof. Consider the enveloping semigroup $E(X, T)=E$. By $1.9 E$ is a compact topological group. But the inclusion $T \rightarrow E$ is a homomorphism of $T$ onto a dense subgroup; therefore $E$ is a compact connected solvable topological group. It follows that $E$ is abelian. If $E$ is a Lie group this is well known, in general $E$ is an inverse limit of quotients of $E$ which are Lie groups.

Now the fact that $E$ is abelian implies that any element $t \in T$ which is in the commutator of $T$ is in the kernel of the inclusion $T \rightarrow E$. Thus $t$ acts as the identity homeomorphism of $X$. 
COROLLARY 2.8. Let T be a connected locally compact solvable topological group which acts effectively and equicontinuously on a compact Hausdorff space $X$. Then Tis abelian.

We end this section with an elementary lemma on realizing transformation groups as suspensions which will be used and generalized in $\$ 4$. (see $\S 5$ ).

LEMMA 2.9. Let $(Z, T)$ be a T-transformation group, and $S$ a closed syndetic subgroup of $T$. Suppose $\varnothing \neq X \subset Z$ satisfies:

(i) $X$ is closed and invariant under $S$,

(ii) if $X t \cap X \neq \varnothing$ then $t \in S$.

Then $(X, S)$ is an $S$-transformation group and $\left(X \times_{S} T, T\right) \cong(Z, T)$.

Proof. The map $X \times{ }_{s} T \rightarrow Z$ given by $[x, t] \rightarrow x t$ is a $T$-isomorphism.

Remark 2.10. (1) The converse of 2.9 clearly holds; indeed the subset

$$
X_{e}=\{[x, e] / x \in X\} \subset X \times T
$$

satisfies conditions (i) and (ii).

(2) If $S$ is contained in the centre of $T$ then 2.9 can be used to show that

$$
(E(X, S) \underset{S}{\times} T, T) \cong(E(X \underset{S}{X}), T) .
$$

This fact can in turn be used to give an alternative proof of 2.4. (3) In general the homomorphism of $S$-transformation groups $E(X, S) \rightarrow X$ induces a homomorphism of $T$-transformation groups

$$
(E(X, S) \underset{S}{\times} T, T) \rightarrow(\underset{S}{(X)} \times \underset{S}{X}, T)
$$

which in turn induces a homomorphism

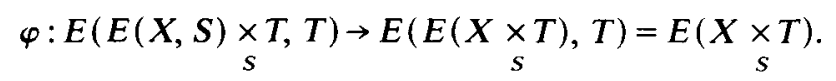

Indeed it is not hard to check that $\varphi$ is an isomorphism.

\section{Continuous transformation groups}

The group of a minimal flow $(X, T)$ has proved to be useful in studying the dynamical properties of $(X, T)$ (see [E1] for example). This group however is independent of the topology on $T$; that is the group of $(X, T)$ is the same as that of $\left(X, T^{\delta}\right)$. In this section we will give an analogous construction of the group of a minimal flow which is sensitive to the topology placed on $T$; this construction reduces to the usual notion when $T$ is given the discrete topology. In particular, since the notion of a syndetic subgroup $S$ of $T$ is heavily dependent on the topology on $T$, these groups prove to be useful in comparing the dynamical properties of an $S$-flow with those of its suspension to a $T$-flow.

The construction of these groups mimics the treatment in [E2]; the proofs of some of their properties are quite similar to those given in [E2] and hence they will often just be outlined. We begin with some notation. Recall that $T$ denotes a locally compact topological group, and $S$ a closed syndetic subgroup of $T$.

Notation 3.1. We will denote by $\beta T$ the Stone-Cech compactification of the discrete topology on $T$ (not the given topology). It is standard that $\beta T$ can be provided 
with a transformation group structure which is pointed by the identity $e \in T \subset \beta T$; in fact $\beta T$ is equipped with a semigroup structure in such a way that

$$
\begin{array}{rlrlrl}
L_{p}: \beta T & \rightarrow \beta T \text { and } & R_{t}: \beta T & \rightarrow \beta T \\
q & \rightarrow p q & q & \rightarrow q t
\end{array}
$$

are continuous for all $p \in \beta T$ and $t \in T$.

It is well known that the transformation group $(\beta T, e)$ is universal for pointed $T$-transformation groups. That is, given a pointed $T$-flow $\left(X, x_{0}\right)$ there exists a unique homomorphism $(\beta T, e) \rightarrow\left(X, x_{0}\right)$. Note however that the action $\beta T \times T \rightarrow \beta T$ is continuous for the discrete topology on $T$ but not necessarily for the given topology.

We denote by $\mathscr{C}=\mathscr{C}(\beta T)=\{f: \beta T \rightarrow \mathbf{R} / f$ is continuous $\}$. Note that $\mathscr{C}$ is a Banach algebra with the supremum norm. If $f \in \mathscr{C}$ we denote the value of $f$ at $p \in \beta T$ by $\langle f, p\rangle$. For $t \in T$ and $p \in \beta T$ the functions $t f, f p \in \mathscr{C}$ are defined by

$$
\begin{array}{cl}
\langle t f, q\rangle=\langle f, q t\rangle & \text { for all } q \in \beta T \\
\langle f p, q\rangle=\langle f, p q\rangle & \text { for all } q \in \beta T .
\end{array}
$$

Let $\mathscr{A} \subset \mathscr{C}(\beta T)$ be a subalgebra, we say that $\mathscr{A}$ is a $T$-subalgebra if

(i) $\mathscr{A}$ is closed,

(ii) $\mathbf{R} \subset \mathscr{A}$ ( $\mathscr{A}$ contains the constant functions),

(iii) if $f \in \mathscr{A}$ then $t f \in \mathscr{A}$ for all $t \in T$.

A generalization of the Stone-Weierstrass theorem implies that the category of $T$-subalgebras of $\mathscr{C}$ with inclusions as morphisms is naturally equivalent to the category of pointed $T$-transformation groups. We will use the notation $|\mathscr{A}|$ for the unique (up to homeomorphism) compact Hausdorff space such that

$$
\mathscr{A} \cong\{f:|\mathscr{A}| \rightarrow \mathbf{R} / f \text { is continuous }\} \stackrel{\text { def }}{=} \mathscr{C}(|\mathscr{A}|) .
$$

We say that $f: T \rightarrow \mathbf{R}$ is right uniformly continuous if $\lim _{t \rightarrow e}\|t f-f\|=0$. We write $\mathfrak{R}_{T}$ for the $T$-subalgebra of $\mathscr{C}$ consisting of all bounded right uniformly continuous functions $f: T \rightarrow \mathbf{R}$. (Any bounded function $f: T \rightarrow \mathbf{R}$ has a unique continuous extension to $\beta T$.) Note that when $T$ is given the discrete topology $\Re_{T^{\delta}}=\mathscr{C}$ and $\left|\Re_{T^{\delta}}\right|=\beta T$.

The next lemma shows that $\left(\left|\Re_{T}\right|, T\right)$ is universal for pointed continuous $T$ transformation groups (the topology on $T$ being taken into account).

Lemma 3.2. Let $T$ be a locally compact topological group and $(X, T)$ a pointed T-transformation group. Then

(1) there exists a unique homomorphism of T-transformation groups $\left(\left|\mathfrak{R}_{T}\right|, T\right) \rightarrow$ $(X, T)$,

(2) $\left|\Re_{T}\right|$ admits a semigroup structure in which $L_{p}$ is continuous for all $p \in\left|\Re_{T}\right|$ and $R_{t}$ is continuous for all $t \in T$; moreover the canonical T-homomorphism $\pi: \beta T \rightarrow$ $\left|\mathfrak{H}_{T}\right|$ is a homomorphism of semigroups,

(3) The natural inclusion $T \rightarrow \beta T \stackrel{\pi}{\rightarrow}\left|\Re_{T}\right|$ gives an isomorphism of $T$ into $\left|\Re_{T}\right|$ with $\bar{T}=\left|\Re_{T}\right|$. 
Comments on Proof. Rather than giving a detailed proof here we remark that (1) is proven in [E2]. It is important to note that the assertion that the action $\left|\Re_{T}\right| \times T \rightarrow\left|\Re_{T}\right|$ is continuous is part of statement (1).

Statement (2) is proven by noting that $\left|\Re_{T}\right|$ has the quotient topology given by the map $\pi: \beta T \rightarrow\left|\Re_{T}\right|$, and defining the semigroup structure on $\left|\Re_{T}\right|$ so as to make $\pi$ a homomorphism of semigroups. Alternatively one can observe that by (1) the $T$-homomorphism $E\left(\left|\mathfrak{M}_{T}\right|, T\right) \rightarrow\left|\Re_{T}\right|$ from the enveloping semigroup of $\left|\Re_{T}\right|$ to $\left|\Re_{T}\right|$ given by evaluation at the base point must be an isomorphism.

Statement (3) is proven by Veech in [V]. (See his Appendix, pp. 823-824.)

LEMMA 3.3. Let $S \subset T$ be a closed syndetic subgroup and denote by $\bar{S}$ the closure of $S$ in $\left|\Re_{T}\right|$. Then

(1) The product map $\bar{S} \times T \rightarrow\left|\Re_{T}\right|$ induces an isomorphism $\left(\bar{S} \times_{S} T, T\right) \rightarrow\left(\left|\Re_{T}\right|, T\right)$ of T-transformation groups.

(2) $(\bar{S}, S)$ is universal for continuous pointed $S$-transformation groups. (Here $S$ is provided with the induced topology).

Proof. (1) $\bar{S}$ is closed in $\left|\Re_{T}\right|$, hence compact. The product map is the restriction of the map $\left|\mathfrak{R}_{r}\right| \times T \rightarrow \mathfrak{R}_{T} \mid$ and hence is continuous by 3.2 . In particular $(\bar{S}, S)$ is a continuous pointed (by $e \in S$ ) $S$-transformation group when $S$ is given the induced topology. Since $S$ is syndetic in $T,\left(\bar{S} \times{ }_{S} T, T\right)$ is a pointed continuous $T$-transformation group. It is straightforward to check that the product map induces a homomorphism; but by $3.2\left(\left|\Re_{r}\right|, T\right)$ is universal, hence it must be an isomorphism.

(2) Now suppose that $\left(X, x_{0}\right)$ is a continuous pointed $S$-transformation group, and consider $\left(X \times_{S} T,\left[x_{0}, e\right]\right)$. Since $\left(\left|\Re_{T}\right|, e\right)$ is universal we obtain a $T$ homomorphism

$$
\psi:\left(\left|\Re_{T}\right|, e\right) \rightarrow\left(\underset{S}{X} T,\left[x_{0}, e\right]\right) .
$$

But then $\psi(\bar{S})=\{[x, e] / x \in X\}$ so the restriction of $\psi$ to $\bar{S}$ induces an $S$ homomorphism $(\bar{S}, e) \rightarrow\left(X, x_{0}\right)$.

Corollary 3.4. Let $S \subset T$ be a closed syndetic subgroup of $T$ and let $\bar{S}$ denote the closure of $S$ in $\left|\Re_{T}\right|$. Then $\bar{S} t \cap \bar{S} \neq \varnothing$ if and only if $t \in S$.

Proof. By 3.3 the map

$$
\varphi: \underset{S}{S} \times \underset{S}{\rightarrow}\left|\Re_{T}\right| \quad \varphi[x, t]=x t
$$

is a $T$-isomorphism. Now suppose that $x \in \bar{S}$ and $x t=y \in \bar{S}$ (i.e. $\bar{S} t \cap \bar{S} \neq \varnothing$ ). Then $\varphi[y, e]=\varphi[x, t]$ and hence $[y, e]=[x, t]$ and $t \in S$. Clearly $t \in S$ implies $\bar{S} t=\bar{S}$.

Notation 3.5. Let $S$ be a closed syndetic subgroup of $T$ and $M$ a minimal right ideal in $\left|\Re_{T}\right|$. We set

$$
M_{S}=M \cap \bar{S}
$$

where as usual $\bar{S}$ is the closure of $S$ in $\left|\mathcal{R}_{T}\right|$.

Remark 3.6. Let $M$ be a minimal right ideal in $\left|\Re_{T}\right|$ and $u \in M$ an idempotent. It is shown in [E2] that $(M, u)$ is a universal continuous pointed minimal $T$-transformation group. 
LEMMA 3.7. Let $S$ be a closed syndetic subgroup of $T, M$ a minimal ideal in $\left|\Re_{T}\right|$, and $u \in M_{S}$ an idempotent. Then $\left(M_{S}, u\right)$ is a universal continuous pointed minimal $S$ transformation group.

Proof. It follows from 3.3 that $(\bar{S}, S) \cong\left(\left|\Re_{S}\right|, S\right)$; hence it suffices to show that $M_{S}$ is a minimal right $\bar{S}$ ideal (by 3.6 with $T$ replaced by $S$ ). Now $M_{S} \bar{S} \subset \bar{S}$ because $\bar{S}$ is a subsemigroup of $\left|\Re_{T}\right|$ and $M_{S}=M \cap \bar{S} \subset \bar{S}$. On the other hand $M_{S} \bar{S} \subset \boldsymbol{M} \bar{S} \subset$ $M\left|\Re_{T}\right|=M$, since $M$ is a right ideal. Thus $M_{S} \bar{S} \subset M \cap \bar{S}=M_{S}$ and $M_{S}$ is a right ideal in $\bar{S}$.

To see that $M_{S}$ is minimal let $p, q \in M_{S}$, we will show that $q \in \overrightarrow{p S}$. But $p, q \in M$ and $M$ is minimal (as a $T$-transformation group) so there exists a net $\left\{t_{n}\right\} \subset T$ such that $p t_{n} \rightarrow q$. Since $S$ is syndetic in $T$ we can write $t_{n}=s_{n} k_{n}$ with $\left\{s_{n}\right\} \subset S$ and $\left\{k_{n}\right\} \subset K$ where $K \subset T$ is compact. Now $\left\{p s_{n}\right\} \subset M_{S}$ which is compact, so by passing to a subnet if necessary we may assume that $p s_{n} \rightarrow \hat{p} \in M_{S}$ and $k_{n} \rightarrow k \in K$. Then $p t_{n}=$ $p s_{n} k_{n} \rightarrow \hat{p} k$ so $\hat{p} k=q$. But $\hat{p}, q \in M_{S} \subset \bar{S}$ hence $\bar{S} k \cap \bar{S} \neq \varnothing$. By 3.4 this implies that $k \in S$. Therefore $q=\hat{p} k \in(\overline{p S}) S=\overline{p S}$ and $M_{S}$ is minimal.

Notation 3.8. Let $S \subset T$ be a closed syndetic subgroup, and $M$ a fixed ideal in $\left|\Re_{T}\right|$. Fix an idempotent $u=u_{S} \in M_{S}=M \cap \bar{S} \subset M$. We set

$$
\begin{gathered}
\mathscr{A}(u)=\left\{f \in \mathfrak{R}_{T} / f u=f\right\} \\
G=M u \text { and } G_{S}=M_{S} u .
\end{gathered}
$$

LEMMA 3.9. Let $S$ be a normal syndetic subgroup of $T$ and $M \subset\left|\Re_{T}\right|$ a minimal ideal. Let $w \in M$ be an idempotent. Then $w \in M_{S}$.

Proof. The $T$-transformation group $(M, T)$ is minimal so $w \in M$ is an almost periodic point of $(M, T)$. But $S$ is normal and syndetic in $T$ so $w$ is an almost periodic point of $(\overline{w S}, S)$; hence $(\overline{w S}, S)$ is a minimal $S$-transformation group (see 2.5 and 2.8 of [E2]).

Now choose an idempotent $v \in M_{S}$. Since $v \in M$ we have $v=w v \in w \bar{S}$ (each $p \in M$ can be written $p=w q$ for some $q \in M$, so $w p=w^{2} q=w q=p$ and $L_{w}$ acts as the identity on $M$ ). But $L_{w}$ is continuous so $w \bar{S}=\overline{w S}$; hence $v \in M_{S} \cap \overline{w S}$. Both $M_{S}$ and $\overline{w S}$ are minimal so this implies that $M_{S}=\overline{w S}$ and in particular $w \in M_{S}$.

Remark 3.10. Lemma 3.9 shows that when studying normal closed syndetic subgroups $S \subset T$ we can fix an idempotent $u \in M$ such that $u \in M_{S}$ for all $S$.

In what follows when the assumption is made that $S$ is normal in $T$ we will use a fixed choice of $u$ independent of $S$. This will be important in $\S 9$.

Lemma 3.11. Let $S, T, u, G, G$ be as in 3.8. Then

(1) $G=\{p \in M / p u=p\}$ is a subgroup of $M$ with identity $u$,

(2) $G_{S}=\left\{p \in M_{S} / p u=p\right\}$ is a subgroup of $G$.

Proof. (1) When $T$ is given the discrete topology $\left|\Re_{T}\right|=\beta T$ and this result is just 3.5 of [E2]. The proof of the general case is completely analogous.

(2) Since $\bar{S}=\left|\Re_{S}\right|$ and $M_{S}$ is an $\bar{S}$ minimal ideal (see 3.7); replacing $T$ by $S$ in (1) shows that $G_{S}$ is a group. But the group structure on $G_{S}$ comes from the semigroup structure on $\bar{S}$, and $\bar{S}$ is a subsemigroup of $\left|\Re_{T}\right|$, therefore $G_{S}$ is a subgroup of $G$. 
Lemma 3.12. Let $\pi: \beta T \rightarrow\left|\Re_{T}\right|$ denote the canonical projection. Then

(1) There exists a minimal ideal $M_{\delta} \subset \beta T$ and an idempotent $u_{\delta} \in M_{\delta}$ such that $\pi\left(M_{\delta}\right)=M$ and $\pi\left(u_{\delta}\right)=u$.

(2) Set $G_{\delta}=M_{\delta} u_{\delta}$. Then $\left.\pi\right|_{G_{\delta}}$ is a group homomorphism with kernel

$$
R_{T}=\left\{\alpha \in G_{\delta} / f \alpha=f \text { for all } f \in \mathfrak{R}_{T} \quad \text { with } f u_{\delta}=f\right\} .
$$

Proof. (1) By $3.2 \pi$ is an epimorphism of semigroups, hence $\pi^{-1}(M)$ is a nonvacuous right ideal in $\beta T$. Let $M_{\delta} \subset \pi^{-1}(M)$ be a minimal right ideal. Then $\pi\left(M_{\delta}\right) \subset M$ is a right ideal, so $\pi\left(M_{\delta}\right)=M$ because $M$ is minimal. Moreover $\pi^{-1}(u) \subset M_{\delta}$ is a nonvacuous subsemigroup of $\beta T$ and thus must contain an idempotent $u_{\delta}$.

(2) The group structure on $G_{\delta}$ comes from the semigroup structure on $\beta T$ so $\left.\pi\right|_{G_{\delta}}$ is a group homomorphism. Clearly

$$
\pi\left(M_{\delta} u_{\delta}\right)=\pi\left(M_{\delta}\right) \pi\left(u_{\delta}\right)=M u=G
$$

To see that $\operatorname{ker} \pi=R_{T}$ consider the restriction $\pi: M_{\delta} \rightarrow M$; it is adjoint to the inclusion of algebras

$$
\pi^{*}: \mathscr{A}(u)=\left\{f \in \mathfrak{R}_{T} / f u=f\right\} \rightarrow \mathscr{A}_{\delta}\left(u_{\delta}\right)=\left\{f \in \mathscr{C} / f u_{\delta}=f\right\} .
$$

It is not hard to check that

$$
\mathscr{A}_{\delta} u_{\delta} \subset \mathfrak{R}_{T}=\mathscr{A}(u) \text {. }
$$

Now suppose that $\alpha=p u_{\delta} \in G_{\delta}$ with $\pi(\alpha)=u$. Let $f \in \mathscr{A}_{\delta}\left(u_{\delta}\right) \subset \mathfrak{\Re _ { T }}$; then $f \alpha=$ $f u_{\delta}=f$ because $\pi(\alpha)=\pi\left(u_{\delta}\right)$ (since $f \in \mathfrak{R}_{T}, \pi(p)=\pi(q)$ implies that $f p=f q$ ). This shows that $\alpha \in R_{T}$ and hence that ker $\pi \subset R_{T}$.

On the other hand suppose that $\alpha \in R_{T}$ and let $f \in \mathscr{A}(u)$. Then

$$
\begin{aligned}
f(\pi(\alpha)) & =(f \circ \pi) a \\
\ddots \quad & \left.=(f \circ \pi) u_{\delta} \text { (because } f \circ \pi \in \mathscr{A}_{\delta}\left(u_{\delta}\right) \subset \mathfrak{R}_{T}\right) \\
\ddots & =f\left(\pi\left(u_{\delta}\right)\right) \\
& =f u .
\end{aligned}
$$

This holds for all $f \in \mathscr{A}(u)=\mathscr{C}(M)$ so $\pi(\alpha)=u$ and hence $\alpha \in \operatorname{ker} \pi$.

Remark 3.13. Using the notation of 3.12 we observe that

$$
\text { (i) } \mathscr{C}\left(M_{\delta}\right)=\mathscr{A}_{\delta}\left(u_{\delta}\right)
$$

and

Note that

$$
\text { (ii) } \mathscr{C}(M)=\mathscr{A}(u)=\mathscr{A}_{\delta}\left(u_{\delta}\right) \cap \mathfrak{R}_{T} \text {. }
$$

$$
f \in \mathscr{A}_{\delta}\left(u_{\delta}\right) \cap \mathfrak{M}_{T} \text { implies that } f=f u_{\delta} \in \mathfrak{M}_{T} u_{\delta}
$$

similarly if $f=g u_{\delta} \in \mathfrak{M} u_{\delta}$ then

$$
f u_{\delta}=g u_{\delta}^{2}=g u_{\delta} \quad \text { and } f \in \mathscr{A}_{\delta}\left(u_{\delta}\right) \cap \mathfrak{H}_{T} .
$$

(One needs to check here that $f \in \mathfrak{H}_{T}$ implies $f p \in \mathfrak{K}_{T}$ for all $p \in \beta T$.) Combining these remarks with (ii) above we obtain

$$
\text { (iii) }\left|\mathfrak{K}_{T} u_{\delta}\right| \cong M
$$

Thus $\left(\left|\Re_{T} u_{\delta}\right|, T\right)$ is a universal continuous pointed minimal $T$-transformation group; hence any continuous pointed minimal $T$-transformation group corresponds to a $T$-subalgebra $\mathscr{A} \subset M_{\tau} u_{\delta}$. We are now ready to define the group of a continuous pointed minimal $T$-transformation group. 
Definition 3.14. Let $S$ be a closed syndetic subgroup of $T$ and $\mathscr{A} \subset \Re_{T} u_{\alpha}$ a $T$ subalgebra. Thus $(|\mathscr{A}|, T)$ is a continuous pointed minimal flow and we define the group of $\mathscr{A}$ by

$$
G(\mathscr{A})=G(|\mathscr{A}|, T)=\{\alpha \in G / f \alpha=f \text { for all } f \in \mathscr{A}\} .
$$

Similarly we define the relative $S$-group of $\mathscr{A}$ by

$$
G_{S}(\mathscr{A})=G_{S}(|\mathscr{A}|, T)=\left\{\alpha \in G_{S} / f \alpha=f \text { for all } f \in \mathscr{A}\right\} .
$$

Here $G$ and $G_{S}$ are defined as in 3.8 .

Remarks 3.15. (1) When $T$ is discrete $\left|\Re_{T}\right|=\beta T$ and we use the notation $G_{\delta}(\mathscr{A})$ for the group of the flow $(|\mathscr{A}|, T)$. This coincides with the definition of the group of a minimal flow given in [E1].

(2) Let $\mathscr{A} \subset \mathfrak{H}_{T} u_{\delta}$ and

$$
\Psi:(M, u) \rightarrow\left(|\mathscr{A}|, x_{\mathscr{A}}\right)
$$

be the classifying map (which is adjoint to the inclusion $\mathscr{A} \subset \Re_{T} u_{\delta}$ ). By definition

$$
\alpha \in G(|\mathscr{A}|, T)
$$

if and only if

$$
\alpha \in M u \text { and } f \alpha=f \text { for all } f \in \mathscr{A}
$$

which holds if and only if

$$
\begin{aligned}
\langle f, \alpha\rangle & =\langle f, \alpha u\rangle \\
\alpha \in M u \quad \text { and } \quad & =\langle f \alpha, u\rangle \\
& =\langle f, u\rangle,
\end{aligned}
$$

which in turn holds if and only if

$$
\alpha \in G \text { and } \Psi(\alpha)=x_{\mathscr{A}} .
$$

Noting that an element $\lim t_{n}=p \in \beta T$ acts on $x \in|\mathscr{A}|$ by $x p=\lim x t_{n}$, and that $\Psi$ is a $T$-homomorphism we obtain:

$$
\begin{aligned}
G(|\not{A}|, T) & =\left\{\alpha \in G / \Psi(\alpha)=x_{\not \alpha A}\right\} \\
& =\left\{\alpha \in G /\left(x_{\mathscr{\alpha}}\right)(\alpha)=x_{\alpha \alpha}\right\} .
\end{aligned}
$$

(3) If $\mathscr{A}=\mathbf{R}$ (the constant functions) then $(|\mathscr{A}|, T)$ is the point flow and $G(\mathscr{A})=G$, $G_{S}(\mathscr{A})=G_{S}, G_{\delta}(\mathscr{A})=G_{\delta}$. (By definition $G_{\delta}=M_{\delta} u_{\delta}$ where $M_{\delta}$ and $u_{\delta}$ are as in 3.12.)

(4) $G_{\delta}\left(\Re_{T} u_{\delta}\right)=G_{\delta}(\mathscr{A}(u))=G_{\delta}(M, T)$ (see 3.13). Moreover by definition

$$
G_{\delta}\left(\Re_{T} u_{\delta}\right)=\left\{\alpha \in G_{\delta} / f \alpha=f \text { for all } f \in \Re_{T} u_{\delta}\right\}
$$

But since $\mathfrak{H}_{T} u_{\delta}=\mathscr{A}_{\delta}\left(u_{\delta}\right) \cap \mathfrak{H}_{T}$ we have, using 3.12, that

$$
G_{\delta}(M, T)=R_{T}=\operatorname{ker}\left\{\pi: G_{\delta} \rightarrow G\right\} .
$$

(5) It is clear from the definitions that $\mathscr{A} \subset \mathscr{B}$ implies $G(\mathscr{B}) \subset G(\mathscr{A}), G_{S}(\mathscr{B}) \subset$ $G_{S}(\mathscr{A})$ and $G_{\delta}(\mathscr{B}) \subset G_{\delta}(\mathscr{A})$. It follows from (3) that

$$
R_{T} \subset G_{\delta}(\mathscr{A}) \text { for every } \mathscr{A} \subset \Re_{T} u_{\delta} .
$$


It then follows from 3.12 that the projection $\pi: G_{\delta} \rightarrow G$ induces an isomorphism of groups

$$
\pi: G_{\delta}(|\mathscr{A}|, T) / R_{T} \stackrel{\cong}{\rightarrow} G(|\mathscr{A}|, T)
$$

for all $\mathscr{A} \subset \mathfrak{H}_{T} u_{\delta}$.

(6) It is possible using the isomorphism in (*) above to prove theorems about how the groups $G(\mathscr{A})$ behave with regard to proximal, distal and almost periodic extensions which are analogous to those proven for $G_{\delta}$ in [E2]. In particular we will make use later on of the fact that if $\mathscr{A} \subset \mathscr{B}$ are distal with $G(\mathscr{A})=G(\mathscr{B})$ then $\mathscr{A}=\mathscr{B}$.

LEMMA 3.16. Let $S$ be a closed syndetic subgroup of $T$, and $\mathscr{A} \subset \mathfrak{R}_{T} u_{\delta}$ a $T$-subalgebra. Then $G_{S}(\mathscr{A})=G(\mathscr{A}) \cap \bar{S}$. (As usual $\bar{S}$ is the closure of $S$ in $\left.\left|\Re_{T}\right|.\right)$

Proof. Recall that by $3.11, G_{S}=M_{S} u$ is a subgroup of $G=M u$. The result follows immediately from 3.15 and the fact that $G_{S}=G \cap \bar{S}$. It is clear that

$$
G_{S}=M_{S} u \subset M_{S}=M \cap \bar{S} \subset \bar{S} \text { and } G_{S}=M_{S} u \subset M u=G \text {. }
$$

On the other hand,

$$
\begin{aligned}
& \text { if } \alpha \in G \cap \bar{S}=M u \cap \bar{S} \\
& \text { then } \alpha \in M \cap \bar{S}=M_{S} \text { and } \alpha u=\alpha
\end{aligned}
$$

$\left(\alpha=\gamma u\right.$ so $\alpha u=\gamma u^{2}=\gamma u=\alpha$ ), therefore $\alpha \in M_{s} u$.

We now prove a proposition which relates the relative $S$-group of a minimal $T$-flow to the group of an $S$-flow.

Proposition 3.17. Let $S \subset T$ be a closed syndetic subgroup and $\mathscr{A} \subset \mathfrak{R}_{T} u_{\delta}$ a $T$ subalgebra. Let $\iota: \beta S \rightarrow \beta T$ denote the natural inclusion and set $\mathscr{A}_{S}=\iota^{*}(\mathscr{A})$. Then

(1) $\mathscr{A}_{S} \subset \mathfrak{R}_{S^{\iota}}{ }^{-1}\left(u_{\delta}\right)$ is an $S$-subalgebra.

(2) ı induces an isomorphism $\left(\left|\mathscr{A}_{S}\right|, S\right) \cong\left(\overline{x_{\mathscr{A}} S}, S\right)$ of S-transformation groups. ( $x_{\mathscr{A}} \in$ $|A|$ is the base point.)

(3) $\iota$ induces an isomorphism $G\left(\left|\mathscr{A}_{S}\right|, S\right) \cong G_{S}(|\mathscr{A}|, T)$.

Proof. (1) Note that $\iota: \beta S \rightarrow \beta T$ is one-one so that $\iota^{-1}\left(u_{\delta}\right) \in \beta S$ is well-defined. One way to see this is to note that the image $\iota(\beta S)$ satisfies the defining properties of the Stone-Cech compactification, hence $\iota: \beta S \rightarrow \iota(\beta S)$ must be a homeomorphism. Now $\iota$ is a homomorphism of $S$-transformation groups; thus since $\mathscr{A}$ is a $T$ subalgebra (hence also an $S$-subalgebra) of $\mathscr{C}(\beta T)$, we see that $\iota^{*}(\mathscr{A})$ is an $S$ subalgebra of $\mathscr{C}(\beta S)$. Moreover if $f \in \mathscr{A}$ then

$$
\begin{aligned}
\left\langle\iota^{*}(f)\left(\iota^{-1}\left(u_{\delta}\right)\right), p\right\rangle & =\left\langle\iota^{*}(f), \iota^{-1}\left(u_{\delta}\right) p\right\rangle \\
& =\left\langle f, u_{\delta} \iota(p)\right\rangle \\
& =\left\langle f u_{\delta}, \iota(p)\right\rangle \\
& =\langle f, \iota(p)\rangle \\
& =\langle\iota *(f), p\rangle \quad \text { for all } p \in \beta S,
\end{aligned}
$$

$\left(f u_{\delta}=f\right.$ because $\mathscr{A} \subset \mathfrak{K}_{T} u_{\delta}$ ). Since $\iota^{*}(f) \in \mathfrak{H}_{S}$ (because it is the restriction of a $T$-right-uniformly-continuous function to $S$ ), this shows that $\iota^{*}(\mathscr{A}) \subset \mathfrak{H}_{S} \iota^{-1}\left(u_{\delta}\right)$. 
(2) The proof of this statement is similar to the proof in the discrete case (which appears in [E2]) and will be left to the reader.

(3) $\iota$ induces $\iota:\left|\Re_{S}\right| \rightarrow\left|\mathscr{R}_{T}\right|$ which must be an $S$-transformation group isomorphism onto $\bar{S} \subset\left|\Re_{T}\right|$ since both are universal pointed continuous $S$-flows. Using $\iota$ to identify $\left(\iota^{-1}\left(M_{S}\right), \iota^{-1}(u)\right)$ with $\left(M_{S}, u\right)$ we see that

$$
G\left(\left|\mathscr{A}_{S}\right|, S\right)=G\left(\left|\iota^{*}(\mathscr{A})\right|, S\right)
$$

is identified with

$$
\left\{\alpha \in M_{S} u / f \alpha=f \text { for all } f \in \mathscr{A}\right\}=G_{S}(|\mathscr{A}|, T) .
$$

\section{Realizing transformation groups as suspensions}

In this section we show that if $S$ is a closed syndetic subgroup of $T$ and $(|\mathscr{A}|, T)$ is a pointed continuous minimal $T$-flow then $|\mathscr{A}| \cong \overline{x_{\mathscr{A}}} \bar{S} \times{ }_{S} T$ if and only if $G(\mathscr{A})=$ $G_{S}(\mathscr{A})$. When $T$ is abelian this enables us to give an interpretation of total minimality for a flow $(|\mathscr{A}|, T)$ in terms of its group $G(\mathscr{A})$.

We begin with a calculation of $G\left(X \times_{S} T, T\right)$ from $G(X, S)$ when $(X, S)$ is a continuous $S$-transformation group.

Proposition 4.1. Let $S$ be a closed syndetic subgroup of $T$ and $(X, T)$ a continuous pointed $S$-minimal flow. Then $G(X, S)=G\left(X \times_{S} T, T\right)$ where we identify $\alpha \in G(X, S)$ with its image under $\iota:\left|\Re_{S}\right| \rightarrow\left|\Re_{T}\right|$. (See 3.17.)

Proof. Let $\mathscr{A}$ be the $S$-subalgebra of $\Re_{S^{l}}{ }^{-1}\left(u_{\delta}\right)$, and $\mathscr{B}$ the $T$-subalgebra of $\Re_{T} u_{\delta}$ corresponding to $(X, S)$ and $\left(X \times_{S} T, T\right)$ respectively. Let $x_{\mathscr{A}} \in|\mathscr{A}|=X$ be the base point; then $\left[x_{s}, e\right] \in X \times_{S} T$ is the base point. Consider the classifying map

$$
\psi:(M, u) \rightarrow\left(X \times_{S} T,\left[x_{s A}, e\right]\right) .
$$

Its restriction to $M_{S}$ gives a classifying map

$$
\bar{\psi}:\left(M_{S}, u\right) \rightarrow\left(X, x_{\mathscr{A}}\right)
$$

where we identify $X$ with $\{[x, e] / x \in X\} \subset X \times_{s} T$. We can identify $\bar{\psi}$ with the classifying map $\hat{\psi}$ using $\iota$ :

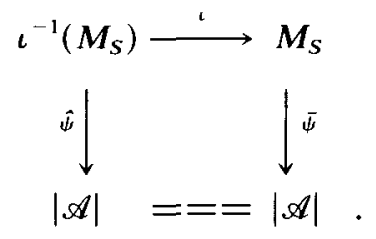

Now by $3.15(2)$

$$
\begin{aligned}
& G(X, S)=\left\{\alpha \in \iota^{-1}\left(M_{S}\right) \iota^{-1}(u) / \hat{\psi}(\alpha)=x_{, \alpha}\right\} \\
& =\left\{\alpha \in M_{S} u / \bar{\psi}(\alpha)=x_{s \mathcal{A}}\right\} \\
& =\left\{\alpha \in M_{S} u / x_{r q} \alpha=x_{. \alpha}\right\},
\end{aligned}
$$

under the identification by $\iota$. Similarly

$$
\begin{aligned}
G(X \times T, T) & =\left\{\alpha \in M u / \psi(\alpha)=\left[x_{s s}, e\right]\right\} \\
& =\left\{\in M u /\left[x_{s}, e\right] \alpha=\left[x_{. \alpha}, e\right]\right\} .
\end{aligned}
$$

With these identifications it is clear that $G(X, S) \subset G\left(X \times_{S} T, T\right)$. 
Now suppose that $\alpha \in G=M u$ with $\left[x_{\mathscr{A}}, e\right] \alpha=\left[x_{\mathscr{A}}, e\right]$. Let $K \subset T$ be a compact subset with $S K=T$. Then

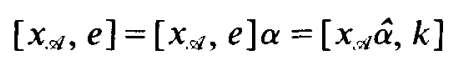

for some $\hat{\alpha} \in M_{S}$ and $k \in K$. But this implies that $k \in S$ and $\alpha=\hat{\alpha} k \in M_{S}$ satisfies $x_{\mathscr{A}} \alpha=x_{\mathscr{A} A}$. The fact that $\alpha u=\alpha$ implies that $\alpha \in M_{S} u=G_{S}$, hence $\alpha \in M_{S} u$ and $G(X, S)=G\left(X \times{ }_{S} T, T\right)$.

Proposition 4.2. Let $S$ be a closed syndetic normal subgroup of $T$ and let $\mathscr{A}$ be a T-subalgebra of $\Re_{T} u_{\delta}$. Set $X=\overline{x_{\mathscr{A}} S}=\left|\mathscr{A}_{S}\right| \subset|\mathscr{A}|$, and set $S_{1}=\{t \in T / X t \cap X \neq \varnothing\}$. Then

(1) $S_{1}=\{t \in T / X t=X\}$ and hence $S_{1}$ is a subgroup of $T$,

(2) there is an action of the compact group $L=S_{1} / S$ on $X \times_{S} T$ such that

$$
\left(X \times_{s} T / L, T\right) \cong(|\mathscr{A}|, T),
$$

(3) the product map $X \times T \rightarrow|\mathscr{A}|$ induces a T-isomorphism

$$
\left(X \times \underset{s_{1}}{\times} T, T\right) \cong(|\mathscr{A}|, T) .
$$

Proof. (1) $\mathscr{A} \subset \mathfrak{K}_{T} u_{\delta}$ so $(|\mathscr{A}|, T)$ is minimal. Since $S$ is syndetic $(X, S)$ is minimal. Now suppose that $t \in T$ with $X t \cap X \neq \varnothing$. Since $S$ is normal in $T, X t$ is invariant and minimal under $S$. Thus $X t \cap X=X=X t$.

(2) Note that $L$ is a group because $S$ is normal in $T$ and $L$ is compact since $S$ is syndetic. Let $S s_{1} \in L$ and set

$$
S s_{1} \cdot[x, t]=\left[x s_{1}^{-1}, s_{1} t\right] \text {. }
$$

It is easy to check that this defines an action of $L$ on $X \times_{s} T$. Now consider the map

$$
X \times T \stackrel{\varphi}{\rightarrow}|\mathscr{A}| \text { given by }[x, t] \rightarrow x t .
$$

It is clear that $\varphi\left(S s_{1} \cdot[x, t]\right)=\varphi[x, t]$ for all $s_{1} \in S_{1}$, thus $\varphi$ induces a map

$$
X \times_{S} T / L \stackrel{\hat{\varphi}}{\rightarrow}|\mathscr{A}| \text {. }
$$

Now suppose that $\hat{\varphi}[x, t]=\hat{\varphi}\left[x_{1}, t_{1}\right]$. Then $x t=x_{1} t_{1}$ and $x=x_{1} t_{1} t^{-1}$; thus $X \cap$ $X t_{1} t^{-1} \neq \varnothing$ and $t_{1} t^{-1} \in S_{1}$. Therefore $S t_{1} t^{-1} \cdot[x, t]=\left[x t t_{1}^{-1}, t_{1} t^{-1} t\right]=\left[x_{1}, t_{1}\right]$ and $\hat{\varphi}$ is an isomorphism of $T$-transformation groups.

(3) The proof of this statement is similar to that of (2) and will be left to the reader.

Remark 4.3. In [E2] Ellis defines the so-called $\tau$-topologies on the group of a minimal flow. In our notation, for each minimal $\mathscr{A}$ there is a topology $\tau(\mathscr{A})$ on $G_{\delta}$ making it compact and making $G_{\delta} / G_{\delta}(\mathscr{A})$ a $T_{1}$ space. We define the $\tau(\mathscr{A})$ topology on $G$ to be the quotient topology under the map

$$
G_{\delta} \rightarrow G_{\delta} / R_{T} \cong G
$$

given in $3.15(5)$.

These topologies are used in the following corollary.

Corollary 4.4. Let $S, T, S_{1}, \mathscr{A}$, and $X$ be as in 4.2. Then $G(\mathscr{A}) / G_{S}(\mathscr{A})$ is homeomorphic to $S_{1} / S$. 
Proof. We first observe that it is shown on p. 146 of [E2] that if $|\mathscr{B}|$ is a compact extension of $|\mathscr{A}|$ by $L$ (i.e. $|\mathscr{B}| / L \cong|\mathscr{A}|$ ) then $L \cong G_{\delta}(\mathscr{A}) / G_{\delta}(\mathscr{B})$ where the latter is given the $\tau(\mathscr{B})$ topology. Applying this to $|\mathscr{B}|=X \times_{S} T$ and $|\mathscr{A}|$ of 4.2 we obtain that

$$
\begin{aligned}
S_{1} / S & \cong G_{\delta}(\mathscr{A}) / G_{\delta}(\mathscr{B}) \\
& \cong G_{\delta}(\mathscr{A}) / R_{T} / G_{\delta}(\mathscr{B}) / R_{T} \\
& \cong G(\mathscr{A}) / G(\mathscr{B}) \\
& \cong G(\mathscr{A}) / G\left(\mathscr{A}_{S}\right)(\text { by } 4.1) \\
& \cong G(\mathscr{A}) / G_{S}(\mathscr{A})(\text { by } 3.17) .
\end{aligned}
$$

As an additional corollary to 4.2 we obtain necessary and sufficient conditions for a $T$-transformation group $(Z, T)$ to be the suspension of $\left(\overline{z_{0} S}, S\right)$. ( $z_{0}$ denotes the base point of $Z$.)

COROLlaRY 4.5. Let $S$ be a closed normal syndetic subgroup of $T, \mathscr{A} \subset \Re_{\tau} u_{\delta}$ a $T$-subalgebra and $X=\left|\mathscr{A}_{S}\right| \subset|\mathscr{A}|$. Then the following are equivalent:

(i) $(|\mathscr{A}|, T) \cong\left(X \times{ }_{S} T, T\right)$,

(ii) $X t \cap X \neq \varnothing$ if and only if $t \in S$,

(iii) $G_{S}(\mathscr{A})=G(\mathscr{A})$,

(iv) $\boldsymbol{G}(\mathscr{A}) \subset \overline{\boldsymbol{S}} \subset\left|\Re_{T}\right|$.

Proof. Using the notation of 4.2 , set $S_{1}=\{t / X t \cap X \neq \varnothing\}$. Then it is clear that (ii) above is equivalent to the statement that $S_{1}=S$, hence 4.2 shows that (ii) implies (i). The fact that (i) implies (ii) is easily checked.

It follows immediately from 4.4 that (ii) is equivalent to (iii). Finally (iii) and (iv) are equivalent since $G_{S}(\mathscr{A})=G(\mathscr{A}) \cap \bar{S}$ by 3.16 .

We conclude this section with a condition on the group of a minimal flow which is equivalent to total minimality when the group $T$ is abelian. Since the condition depends only on the group of the flow it shows in particular that total minimality is a proximal invariant. First we recall the definition of total minimality.

Definition 4.6. Let $(X, T)$ be a pointed minimal $T$-transformation group with base point $x_{0}$. We say that $(X, T)$ is totally minimal if $\overline{x_{0} S}=X$ for all normal closed syndetic subgroups $S \subset T$. Note that this is equivalent to the statement that $\overline{x S}=X$ for all $x \in X$ and $S$ a normal closed syndetic subgroup of $T$.

Proposition 4.7. Let $T$ be abelian and $\mathscr{A} \subset \mathfrak{M}_{T} u_{\delta}$ a $T$-subalgebra. Then the following are equivalent:

(i) $(|\mathscr{A}|, T)$ is totally minimal,

(ii) if $S \subset T$ is closed and syndetic then $G(\mathscr{A}) / G_{S}(\mathscr{A}) \cong T / S$,

(iii) if $S \subset T$ is closed and syndetic and $G_{S}(\mathscr{A})=G(\mathscr{A})$ then $S=T$.

Proof. (i) $\Rightarrow$ (ii). Suppose $(|\mathscr{A}|, T)$ is totally minimal and let $S \subset T$ be closed and syndetic. Then in the notation of 4.2 we have $X=\left|\mathscr{A}_{S}\right|=|\mathscr{A}|$ so that the subgroup $S_{1}=\{t / X t \cap X \neq \varnothing\}$ is equal to $T$; therefore by $4.4 G(\mathscr{A}) / G_{S}(\mathscr{A}) \cong T / S$.

(ii) $\Rightarrow$ (iii) is clear. 
(iii) $\Rightarrow$ (i). Assume that (iii) holds and let $S$ be a closed syndetic subgroup of $T$. ( $S$ is normal because $T$ is abelian.) Let $X=\overline{x_{\mathscr{A}} S}=\left|\mathscr{A}_{S}\right|$ and define $S_{1}$ as above. Then $S_{1}$ is a normal syndetic subgroup of $T$ with $X S_{1}=X$ and

$$
(|\mathscr{A}|, T) \cong\left(\underset{S_{1}}{X \times T, T) \quad(\text { by } 4.2) .}\right.
$$

Thus

$$
\begin{aligned}
G(|\mathscr{A}|, T) & \cong G\left(X \times{ }_{S_{1}}, T, T\right) \\
& \cong G\left(X, S_{1}\right) \quad(\text { by } 4.1) \\
& =G\left(\left|\mathscr{A}_{S_{1}}\right|, S_{1}\right) \\
& =G_{S_{1}}(\mathscr{A}) \quad(\text { by } 3.17)
\end{aligned}
$$

and therefore by our assumption (iii) we must have that $S_{1}=T$; hence $X T=X$ and $X=|\mathscr{A}|$. Therefore $(|\mathscr{A}|, T)$ is totally minimal.

\section{Suspensions of equicontinuous flows}

In this section we apply the results of the previous sections to develop a condition under which the suspension of a pointed equicontinuous flow is equicontinuous. We observe that in 4.4 we saw that when $S$ is contained in the center of $T$ and $(X, S)$ is equicontinuous then $\left(X \times{ }_{S} T, T\right)$ is equicontinuous. Here we will obtain a condition for minimal flows in terms of the groups studied in $\S 3$ which is sharp in the sense that if it is violated then $\left(X \times_{S} T, T\right)$ will be non-equicontinuous for some equicontinuous minimal flow $(X, S)$.

We begin with the notion of the maximal equicontinuous $T$-transformation group (tbe so called Bohr compactification of $T$.)

Definition 5.1. Let $T$ be a locally compact topological group. The Bohr compactification $B(T)$ is a compact topological group which satisfies:

(i) there exists a continuous homomorphism $\gamma: T \rightarrow B(T)$ such that $\overline{\gamma(T)}=B(T)$ and

(ii) if $\varphi: T \rightarrow H$ is a continuous homomorphism of $T$ into a compact topological group with $\overline{\varphi(T)}=B(T)$ then $\varphi$ has a continuous extension to a homomorphism $B(T) \rightarrow H$.

LeMmA 5.2. Let $T$ be a locally compact topological group. Then

(1) Tacts on $B(T)$ making $(B(T), T)$ a pointed equicontinuous transformation group.

(2) If $(X, T)$ is an equicontinuous pointed transformation group then $\left(X, x_{0}\right)$ is a factor of $(B(T), e)$.

Proof. This is standard; we leave it to the reader.

Proposition 5.3. Let $S \subset T$ be a closed syndetic normal subgroup. Then the following are equivalent:

(i) the map $j: B(S) \rightarrow B(T)$ induced by $S \rightarrow T$ is one-one

(ii) $B(S) \times_{s} T \cong B(T)$ as $T$-transformation groups

(iii) $G(B(S), S) \cong G(B(T), T)$. 
Proof. (i) $\Leftrightarrow$ (ii). Consider the following diagram:

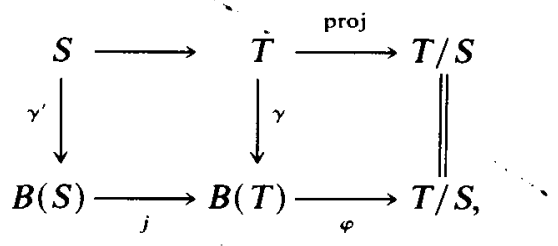

where $\gamma^{\prime}$ and $\gamma$ are the continuous homomorphisms guaranteed by 5.1 , and $\varphi$ is the unique $T$-homomorphism guaranteed by 5.2 and the fact that $(T / S, T)$ is equicontinuous (since $S$ is syndetic). The diagram is commutative by the definition of $j$ and because $T$-homomorphisms are unique. Now the composite

$$
\varphi \circ j \circ \gamma^{\prime}: S \rightarrow T / S
$$

is constant; but $\varphi \circ j$ is continuous and $\overline{\gamma^{\prime}(S)}=B(S)$, therefore $\varphi \circ j$ is constant. It follows that if $\gamma(t) \in j(B(S))$ then $t \in S$ and $\gamma(t)=j\left(\gamma^{\prime}(t)\right)$.

Now the map

$$
\begin{aligned}
B(S) \times T & \rightarrow B(T) \\
(b, t) & \rightarrow j(b) \gamma(t)
\end{aligned}
$$

induces a homomorphism of pointed transformation groups

$$
\psi:(B(S) \underset{S}{\times} T,[e, e]) \rightarrow(B(T), e),
$$

(this follows from the commutativity of the above diagram). Also $\psi\left[b_{1}, t_{1}\right]=\psi\left[b_{2}, t_{2}\right]$ if and only if $j\left(b_{1}^{-1} b_{2}\right)=\gamma\left(t_{1} t_{2}^{-1}\right)$. But by the above this is equivalent to

$$
e=j\left(b_{1}^{-1} b_{2}\right) j\left(\gamma^{\prime}\left(t_{2} t_{1}^{-1}\right)\right)=j\left(b_{1}^{-1} b_{2} \gamma^{\prime}\left(t_{2} t_{1}^{-1}\right)\right) .
$$

Thus $\psi$ is an isomorphism of $T$-transformation groups if and only if $j$ is one-one.

(ii) $\Rightarrow$ (iii). This follows directly from 4.1 .

(iii) $\Rightarrow$ (ii). Suppose that $G(B(S), S) \cong G(B(T), T)$. Then by 4.1

$$
G(B(S) \times T, T)=G(B(T), T) .
$$

Since $B(S)$ is equicontinuous it is distal, hence by $2.3,\left(B(S) \times{ }_{S} T, T\right)$ is distal. But $B(T)$ is a factor of $B(S) \times{ }_{S} T$ so it follows from $3.15(6)$ that $B(S) \times{ }_{S} T \cong B(T)$.

Definition 5.4. Let $T$ be a topological group. A function $f \in \mathscr{C}(\beta T)$ is said to be equicontinuous if $\{t f / t \in T\}$ is relatively compact in $\mathscr{C}(\beta T)$. We denote by $\mathscr{C}=\mathscr{C}_{T}$ the $T$-subalgebra of equicontinuous functions. Note that $\mathscr{E}$ is independent of the topology on $T$.

LEMmA 5.5. Let $T$ be a locally compact topological group. Then $(B(T), T) \cong$ $\left(\left|\mathscr{E}_{T} \cap \mathfrak{H}_{T}\right|, T\right)$.

Proof. When $T$ has the discrete topology this is done in [E2]. That is $\left(B\left(T^{\delta}\right), T^{\delta}\right) \cong$ $\left(\left|\mathscr{E}_{T}\right|, T^{\delta}\right)$. In general if $\mathscr{A} \subset \mathfrak{M}_{T}$ is equicontinuous as a $T$-transformation group then it is equicontinuous as a $T^{\delta}$-transformation group. Thus using 5.2(2) with the discrete topology on $T$, we see that $\left(|\mathscr{A}|, T^{\delta}\right)$ is a factor of $\left(\left|\mathscr{E}_{T}\right|, T^{\delta}\right)$. But then $(|\mathscr{A}|, T)$ is a factor of $\left(\left|\mathscr{E}_{T} \cap \mathfrak{H}_{T}\right|, T\right)$ because $\mathscr{A} \subset \mathfrak{H}_{T}$. Hence $\left(\left|\mathscr{E}_{T} \cap \mathfrak{H}_{T}\right|, T\right)$ is a maximal 
equicontinuous $T$-transformation group. It follows that $\left|\mathscr{E}_{T} \cap \mathfrak{R}_{T}\right|$ is a compact topological group (it must be isomorphic to its enveloping semigroup) which satisfies the conditions of 5.1. Therefore $B(T) \cong\left|\mathscr{E}_{T} \cap \mathfrak{R}_{T}\right|$ as topological groups and hence as $T$-transformation groups.

Proposition 5.6. Let $S$ be a closed normal syndetic subgroup of $T$, with $G\left(\mathscr{E}_{S} \cap \mathfrak{R}_{S}\right) \cong$ $G\left(\mathscr{C}_{T} \cap \mathfrak{R}_{T}\right)$. Let $(X, S)$ be a pointed equicontinuous $S$-transformation group. Then $\left(X \times{ }_{S} T, T\right)$ is equicontinuous.

Proof. By $5.2(X, S)$ is a factor of $(B(S), S)$, thus $\left(X \times_{S} T, T\right)$ is a factor of $\left(B(S) \times{ }_{S} T, T\right)$. On the other hand combining 5.3 with 5.5 we see that $\left(B(S) \times_{S} T, T\right) \cong(B(T), T)$ which is equicontinuous. Therefore $\left(X \times_{S} T, T\right)$ is equicontinuous.

Remark 5.7. If $G\left(\mathscr{E}_{S} \cap \mathfrak{R}_{S}\right) \not \equiv G\left(\mathscr{E}_{T} \cap \Re_{T}\right)$ then by $5.3 B(S) \times_{S} T$ is a nontrivial extension of $B(T)$ and hence by 5.2 cannot be equicontinuous. Note further that in view of 2.5, when $T$ is discrete $G\left(\mathscr{E}_{S} \cap \mathfrak{R}_{S}\right) \cong G\left(\mathscr{E}_{T} \cap \Re_{T}\right)$ for all syndetic normal subgroups $S$ of $T$. In general, 2.4 implies that $G\left(\mathscr{E}_{S} \cap \mathfrak{R}_{S}\right) \cong G\left(\mathscr{E}_{T} \cap \Re_{T}\right)$ when $S$ is a syndetic subgroup of $T$ which is contained in the centre of $T$.

\section{REFERENCES}

[E1] R. Ellis. Group-like extensions of minimal sets. Trans. Amer. Math. Soc. 127 (1967), 125-135.

[E2] R. Ellis. Lectures on Topological Dynamics. Benjamin: New York, 1969.

[Mc-I-W] D. McMahon, E. Ihrig \& T-S Wu. Obstructions to effective equicontinuous and distal actions. Math. Sci. Res. Inst., Berkeley, CA (1983) MSRI 001-84.

[V] W. A. Veech. Topological dynamics. Bull. Amer. Math. Soc. 83 (1977), 775-830. 